\title{
Search for exotic VZ resonances decaying into a jet and dileptons with CMS
}

\author{
Flavia A. Dias* \\ On behalf of the CMS Collaboration \\ Instituto de Física Teórica, Universidade Estadual Paulista \\ E-mail: flavia.dias@cern.ch
}

\begin{abstract}
A search for exotic resonances is performed, with a new particle decaying to a pair of vector bosons $\mathrm{VZ}$, where $\mathrm{V}$ is either a $\mathrm{W}$ or a $\mathrm{Z}$ boson decaying into two overlapping jets and the $\mathrm{Z}$ decays to dielectrons or dimuons. The analysis uses a dataset of proton-proton collisions at $\sqrt{s}=7$ $\mathrm{TeV}$ of center of mass energy, corresponding to $5.0 \mathrm{fb}^{-1}$ of integrated luminosity collected by the Compact Muon Solenoid experiment at the Large Hadron Collider in 2011. No significant excess compared to the standard model background expectation is observed in the mass distribution of the VZ candidates. Upper limits at $95 \%$ confidence level were set on the production cross section times the branching fraction of hypothetical particles decaying to a pair of vector bosons, as a function of the mass. The limits were interpreted in two benchmark models. Sequential Standard Model $\mathrm{W}^{\prime}$ bosons were excluded in the mass range $700-929 \mathrm{GeV}$. Graviton resonances in the Randall-Sundrum model, with $k / \bar{M}_{P l}=0.05$, were excluded in mass range $700-924 \mathrm{GeV}$.
\end{abstract}

36th International Conference on High Energy Physics,

July 4-11, 2012

Melbourne, Australia

${ }^{*}$ Speaker. 


\section{Introduction}

The main reason to search for $\mathrm{VV}$ resonances at high masses has been historically connected to electroweak symmetry breaking models. However, the discovery of a standard model (SM) Higgs boson at $M_{H}=125 \mathrm{GeV} / \mathrm{c}^{2}$ would not exclude all the models being considered - it is possible to accommodate this resonance within the theoretical framework.

The electroweak symmetry breaking mechanism is one of the most fundamental questions being addressed at the LHC. The confirmation of the SM Higgs discovery - the last missing piece of the SM - would validate the spontaneous symmetry breaking via the Higgs mechanism as responsible for the mass generation. However, in many extensions of the SM a spontaneous breaking of the electroweak symmetry is associated with new strong dynamics appearing at the TeV scale. The origin of the new dynamics can be due to new interactions, compact extra dimensions, composite nature of the Higgs boson, or modified models where the Higgs have anomalous couplings.

A search for new heavy resonances decaying to vector boson $\mathrm{V}(\mathrm{V}=\mathrm{W}, \mathrm{Z})$ pairs is performed [1], using $5 \mathrm{fb}^{-1}$ of $\sqrt{s}=7 \mathrm{TeV}$ center-of-mass energy pp collisions data, collected by the CMS experiment [2] in 2011. One $\mathrm{V}$ boson is a $\mathrm{Z} \rightarrow \ell^{+} \ell^{-}(\ell=\mu, e)$, and the other decays hadronically, i.e. $\mathrm{V} \rightarrow q \bar{q}$, with two outgoing quarks overlapping into a single massive jet. The signal is characterized as a peak in the invariant mass of the VZ system, and results are presented in terms of two benchmark scenarios: a Randall-Sundrum (RS) graviton decaying to ZZ and the Sequential Standard Model (SSM) in which a new gauge boson $\mathrm{W}^{\prime}$ with the same couplings as the SM W boson decays to a WZ pair. In both scenarios, the search considers resonances heavier than $700 \mathrm{GeV} / \mathrm{c}^{2}$, where the boosted topology becomes relevant.

\section{Background Estimation}

Muons are reconstructed with a high- $p_{T}$ optimized algorithm [3], and required to have $p_{T}^{\mu}>$ $45 \mathrm{GeV} / \mathrm{c}$ and $|\eta|<2.4^{1}$. Electrons are reconstructed using the Gaussian-sum filter algorithm [4], with $p_{T}^{e}>45 \mathrm{GeV} / \mathrm{c}$ and $|\eta|<2.5$. Particle-flow jets are reconstructed using the infrared-safe anti$\mathrm{k}_{\mathrm{T}}$ algorithm [5] with a distance parameter of 0.7 , and jets are required to have $p_{T}^{\text {jet }}>30 \mathrm{GeV} / \mathrm{c}$ and $|\eta|<2.4$.

Candidate events need to have at least two good quality reconstructed leptons and one jet. Whenever two same-flavor, opposite-sign leptons are found, a $\mathrm{Z}$ candidate is formed summing the four-momenta of the leptons. The selected $\mathrm{Z}$ candidate is required to have invariant mass in the $70<M_{Z}<110 \mathrm{GeV} / \mathrm{c}^{2}$ mass range and with a transverse momentum $p_{T}^{Z}>150 \mathrm{GeV} / \mathrm{c}$. The $\mathrm{V} \rightarrow$ $q \bar{q}$ candidate is reconstructed as a single jet, satisfying $\Delta R$ (jet, $\ell)>1.0^{2}$, where $\ell$ is any of the two leptons forming the $\mathrm{Z}$ candidate; $65<M_{j}<120 \mathrm{GeV} / \mathrm{c}^{2}$, where $M_{j}$ is the jet mass; $p_{T}^{V}>250$ $\mathrm{GeV} / \mathrm{c}$. Once the $\mathrm{Z} \rightarrow \ell^{+} \ell^{-}$and mono-jet $\mathrm{V} \rightarrow q \bar{q}$ candidates have been reconstructed, their four momenta are combined to compute the mass of the mother particle, $M_{V Z}$. This variable is used to evaluate the hypothesis of the signal presence in the analyzed dataset.

According to simulation, the dominant $(\sim 90 \%)$ background after the selection cuts is the inclusive $\mathrm{Z}$ production $(\mathrm{Z}+$ jets), with additional contribution of $t \bar{t}+$ jets and the continuum SM two-

\footnotetext{
${ }^{1} \eta$ is the pseudorapidity, defined as $\eta=-\ln [\tan (\theta / 2)]$

${ }^{2} \Delta R$ is defined as $\Delta R=\sqrt{\Delta \phi^{2}+\Delta \eta^{2}}$
} 
vector boson production (WZ and ZZ). The shape and normalization of the expected background $M_{V Z}$ distributions are derived from data, with additional cross-checks carried out with the inclusive simulated background samples.

The data-driven background estimation uses the product of the $M_{V Z}$ distribution with sideband $M_{j}$ selection $\left(30<M_{j}<65 \mathrm{GeV} / \mathrm{c}^{2}\right)$ and $\alpha\left(M_{V Z}\right)$, the Monte-Carlo ratio of background events in nominal and sideband regions. The derived distribution is then fitted to an analytical function,

$$
f_{A}\left(M_{V Z}\right)=p_{0} \frac{\left[1-\left(\frac{M_{V Z}}{\sqrt{s}}\right)\right]^{p_{1}}}{\left(\frac{M_{V Z}}{\sqrt{s}}\right)^{p_{2}+p_{3} \log \left(\mathrm{M}_{\mathrm{vZ} / \sqrt{\mathrm{s}})}\right.}}
$$

where $\sqrt{s}$ is the collision energy, $p_{0}, \ldots, p_{3}$ are free parameters, and $M_{V Z}$ is expressed in $\mathrm{GeV} / \mathrm{c}^{2}$. The fit determines both the shape and the normalization of the expected background as a function of the $M_{V Z}$ spectrum in the electron and muon channels. The comparisons of the estimated background with the prediction from the simulation and the data $M_{V Z}$ distributions are shown in Fig. 1.
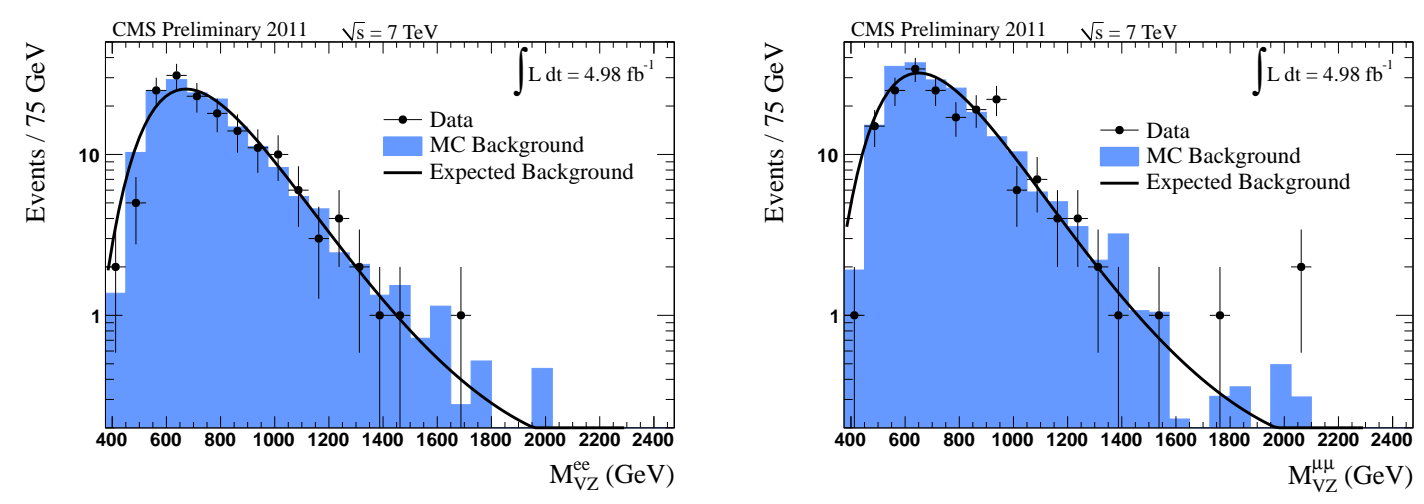

Figure 1: The comparison of the estimated background (black curve) with the total MC background (blue histogram) and the data (black points) $M_{V Z}$ distributions for the electron (left) and the muon (right) channels.

The systematic uncertainties considered can be divided in two categories: uncertainties in the determination of the expected SM background and in the expected yields of signal events. For the signal, the systematic uncertainties considered are the luminosity, jet energy scale, parton distribution functions, reconstruction efficiencies and V mass cut efficiency. For the background, we consider the jet energy scale, the fit variations and the deviation from MC predictions.

\section{Results}

The modified frequentist $\mathrm{CL}_{\mathrm{S}}$ statistical method [6] is employed to search for exotic VZ resonances, and a series of search windows corresponding to different signal mass hypotheses. Each mass window is optimized to give the best exclusion limit, a procedure which is also adequate for establishing new resonance discovery. No significant excess over the expected background is observed. 
The 95\% confidence level exclusion limits on the combined $\sigma(\mathrm{VZ}) \times \mathscr{B}\left(\mathrm{V} \rightarrow q \bar{q}, \mathrm{Z} \rightarrow \ell^{+} \ell^{-}\right.$, $\ell=e, \mu)$ product for the two final states under study are calculated as a function of the mass of the hypothetical resonance. These exclusion limits are interpreted in two benchmark models: Sequential Standard Model $\mathrm{W}^{\prime}$ and RS graviton.

The exclusion limits as a function of the VZ resonance mass can be seen in Fig. 2, where a linear interpolation is used between the benchmark mass values. SSM W' bosons with masses between 700 and $938 \mathrm{GeV} / \mathrm{c}^{2}$ are excluded at $95 \% \mathrm{CL}$. RS gravitons with masses between 750 and $933 \mathrm{GeV} / \mathrm{c}^{2}$ are also excluded, for $k / \bar{M}_{P l}=0.05$. Assuming the resonance width is much smaller than the experimental resolution for the range of $k / \bar{M}_{P l}$ considered, the limit can be extended in the $m_{G}-k / \bar{M}_{P l}$ plane. The extension uses the quadratic dependence of the cross section on $k / \bar{M}_{P l}$, and assumes that the signal efficiency remains the same. The result is shown in Fig. 3.
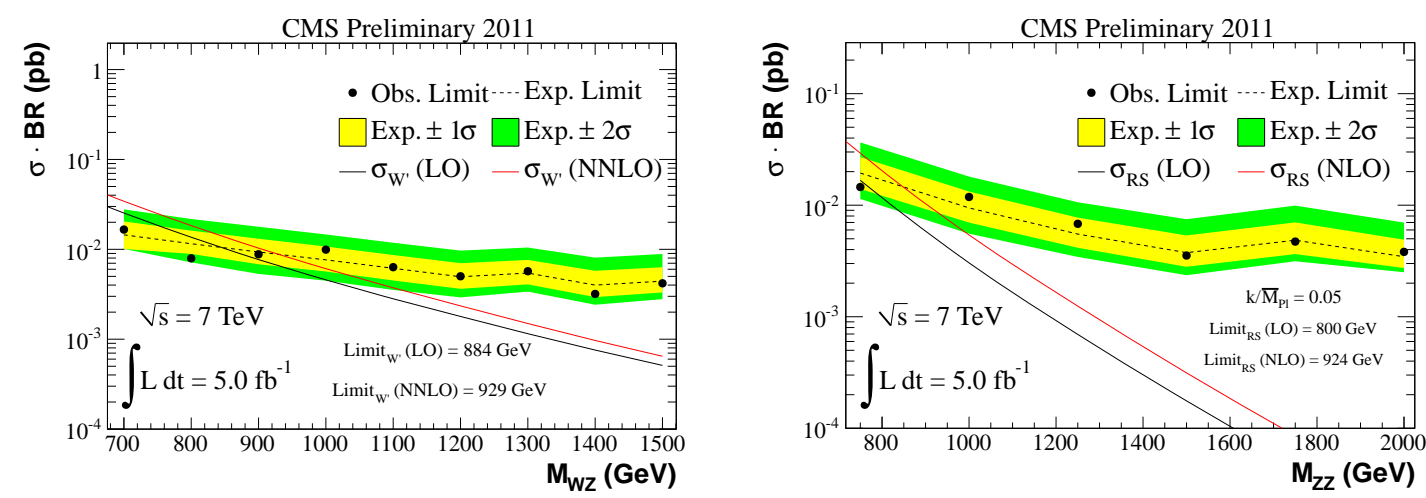

Figure 2: Observed and expected 95\% CL exclusion limits for a counting experiment and comparison with the theoretical predictions in $\mathrm{W}^{\prime}$ (left) and RS Graviton (right) models for the combination of electron and muon channels. The limits are calculated with the frequentist CLs statistical method.

\section{Summary}

A search for exotic resonances was performed, with a new particle decaying to a pair of vector bosons $\mathrm{VZ}$, where $\mathrm{V}$ is either a $\mathrm{W}$ or a $\mathrm{Z}$ boson decaying into two overlapping jets and the $\mathrm{Z}$ decays to dielectrons or dimuons. The analysis uses a dataset of proton-proton collisions at $\sqrt{s}=7 \mathrm{TeV}$ of center of mass energy, corresponding to $5.0 \mathrm{fb}^{-1}$ of integrated luminosity collected by the CMS experiment at the LHC in 2011. No significant excess was observed in the mass distribution of the VZ candidates compared to the background expectation from the processes of the standard model. Lower bounds at $95 \%$ confidence level were set on the mass of the hypothetical particle decaying to the VZ final state in two benchmark models. In Randall-Sundrum model, graviton resonances with masses between 700 and $924 \mathrm{GeV} / \mathrm{c}^{2}$ for $k / \bar{M}_{P l}=0.05$ were excluded. The possible existence of heavy charged vector bosons was also investigated, as the ones predicted by the Sequential Standard Model, and $\mathrm{W}^{\prime}$ bosons were excluded in the mass interval between 700 and $929 \mathrm{GeV} / \mathrm{c}^{2}$. 


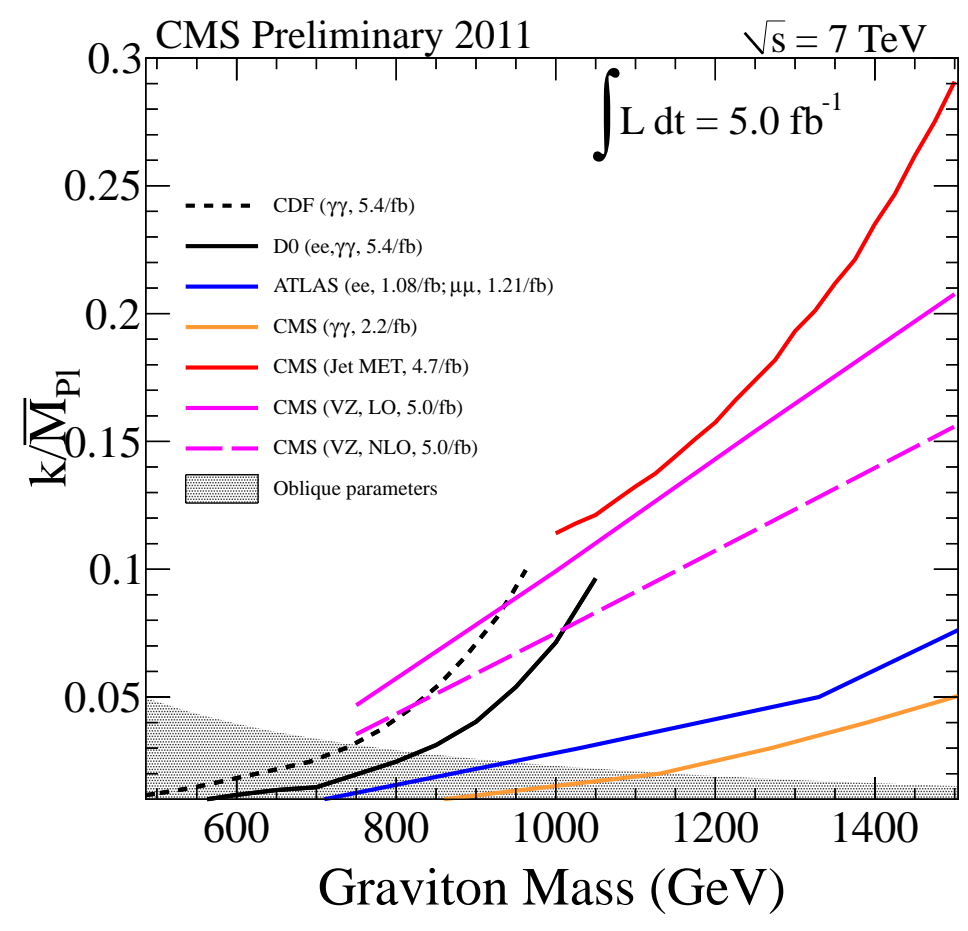

Figure 3: Observed and expected 95\% CL exclusion limits for a counting experiment and comparison with the theoretical predictions in RS Graviton models for the combination of electron and muon channels. The limits are calculated with the frequentist CLs statistical method. The resonance of the hypothetical particle is considered narrow with respect to the experimental resolution.

\section{References}

[1] CMS Collaboration, "Search for exotic resonances decaying into V+Z using final states with a jet and a lepton pair", CMS Physics Analysis Summary EXO-11-081 (2012).

[2] CMS Collaboration, "The CMS experiment at the CERN LHC”, JINST 3 S08004 (2008).

[3] CMS Collaboration, "Performance of muon identification in 2010 data", CMS Physics Analysis Summary MUO-10-004 (2011).

[4] W. Adam, R. Fruhwirth, A. Strandlie et al., "Reconstruction of electrons with the Gaussiam-sum filter in the CMS tracker at the LHC”, ECONF C0303241 (2003).

[5] M. Cacciari, G. P. Salam, and G. Soyez, “The Anti-k(t) jet clustering algorithm”, JHEP 04 (2008).

[6] T. Junk, "Confidence level computation for combining searches with small statistics", Nuclear Instruments Methods in Physics Research Section A: Accelerators, Spectrometers, Detectors and Associated Equipment 434 (1999). 